\title{
The Georgi algorithms of jet clustering
}

\author{
Shao-Feng Ge \\ Max-Planck-Institut für Kernphysik, \\ Heidelberg 69117, Germany \\ KEK Theory Center, \\ Tsukuba 305-0801, Japan \\ E-mail: gesf02@gmail.com
}

ABSTRACT: We reveal the direct link between the jet clustering algorithms recently proposed by Howard Georgi and parton shower kinematics, providing firm foundation from the theoretical side. The kinematics of this class of elegant algorithms is explored systematically for partons with arbitrary masses and the jet function is generalized to $J_{\beta}^{(n)}$ with a jet function index $n$ in order to achieve more degrees of freedom. Based on three basic requirements that, the result of jet clustering is process-independent and hence logically consistent, for softer subjets the inclusion cone is larger to conform with the fact that parton shower tends to emit softer partons at earlier stage with larger opening angle, and that the cone size cannot be too large in order to avoid mixing up neighbor jets, we derive constraints on the jet function parameter $\beta$ and index $n$ which are closely related to cone size cutoff. Finally, we discuss how jet function values can be made invariant under Lorentz boost.

KEYWORDS: Jets

ARXIV EPRINT: 1408.3823 


\section{Contents}

1 Introduction 1

2 Connection with parton shower 2

3 Clustering with massive subjets 4

3.1 The inclusion cone 5

$\begin{array}{ll}3.2 & \text { The exclusion cone }\end{array}$

4 Generalized jet function $\quad 8$

5 Lorentz boost invariance 11

6 Conclusion 12

\section{Introduction}

Due to confinement, partons can not be observed directly. The high-energy partons produced in hard scattering experience shower process first, splitting into low-energy partons which further fragment into low-energy hadrons. Then, the information of hadrons is experimentally measured directly, with the information at the parton level buried in sprays of hadrons and needs to be reconstructed. Jet is a very useful tool for this purpose [1] and various algorithms have been proposed. The list includes the longitudinally invariant $k_{t}$ algorithm [2, 3], the Cambridge/Aachen (C/A) algorithm [4-6], the anti- $k_{t}$ algorithm [7], and the Durham algorithm [8], with different features. For details, please also refer to comprehensive reviews [9-13]. All these jet definitions have a pair-wise feature since the criterion whether two jets should be merged into a single one is based on the distance between them. Only with two jets, a distance can be defined. The difference between different algorithms is basically the away of evaluating the distance.

Recently, a new class of algorithms have been proposed [14] by Howard Georgi. In these Georgi algorithms of jet clustering, a jet function is defined in terms of the jet momentum $P_{\alpha}=\left(E_{\alpha}, \mathbf{P}_{\alpha}\right) \equiv \sum_{i \in \alpha} p_{i}$ as,

$$
J_{\beta}\left(P_{\alpha}\right) \equiv E_{\alpha}-\beta \frac{P_{\alpha}^{2}}{E_{\alpha}}=E_{\alpha}\left(1-\beta \frac{P_{\alpha}^{2}}{E_{\alpha}^{2}}\right),
$$

motivated by the observation that jet emerging leads to fast increase in energy $E_{\alpha}$ and slow increase in the jet mass $P_{\alpha}^{2}$, which is small in the first place. When clustering, the jet function should increase with jets merged, which serves as the criterion of clustering jet in the Georgi algorithms. Note that $\alpha$ is a set of parton indices while $\beta>1$ is a jet 
function parameter. As elaborated in [14], $\beta$ is closely related to the jet cone size cutoff. Its value is of the same place as the distance threshold/cutoff in the traditional pair-wise jet algorithms.

Different from distance between jets, jet function can be applied on a single jet, providing a new feature of evaluating jet globally. With pair-wise jet distance, which can be defined and evaluated locally, the clustering procedure starts from individual hadrons/subjets, merging the closest pair to form a new jet. This merging process iterates until the distance between any pair of jets is larger than the threshold/cutoff. Since it starts from individual hadrons, which can be seen as low-level information, the traditional jet algorithms follow a bottom-up approach. On the other hand, the Georgi algorithms can be implemented in a global way, starting from dividing the $4 \pi$ solid angle into fiducial regions [14]. The jet in each fiducial region can be found by looking only at the fiducial region plus some border region. A subjet should be isolated if without it the jet function becomes larger. This is a top-down approach.

Although not revealed in [14], it should be noted that, the jet function method can also be carried out locally in a bottom-up approach. Starting from evaluating jet function for each individual hadrons, a pair of two subjets should be merged into a single jet if the value of jet function increases when doing so. We can choose the pair with the fastest/slowest increase in jet function to cluster at each step.

In this paper, we first make connection between the jet function (1.1) and the parton shower kinematics to establish a theoretical foundation for the Georgi algorithm in section 2. In [14], the kinematic properties of the Georgi algorithms have been explored analytically for massless partons. We try to generalize the results to the massive case in section 3 and provide further generalization of the jet function definition (1.1) in section 4, to achieve more degrees of freedom. At the end, we briefly discuss how the jet function behaves under Lorentz boost in section 5 and conclude in section 6 .

\section{Connection with parton shower}

The basic motivation behind the definition (1.1) of the jet function $J_{\beta}\left(P_{\alpha}\right)$ is the observation that, jet clustering tends to increase the jet energy $E_{\alpha}$ but the jet mass term $P_{\alpha}^{2} / E_{\alpha}$ does not increase that much. Nevertheless, this key point is not elaborated in [14], by assuming that "combining a collection of lines into a single jet, hence increasing the jet energy, if doing so does not increase the jet mass to much". We will try to establish a direct connection between the jet function $J_{\beta}$ as defined in (1.1) and the kinematics of parton shower, illustrating that the basic motivation of $J_{\beta}$ has sound theoretical foundation.

For both massless [15] and massive [16] parton shower schemes, virtuality can be reconstructed iteratively,

$$
P_{\alpha}^{2}-m_{\alpha}^{2}=\frac{p_{j}^{2}-m_{j}^{2}}{z}+\frac{P_{\alpha-j}^{2}-m_{\alpha-j}^{2}}{1-z}+z(1-z) t,
$$

where $P_{\alpha-j} \equiv P_{\alpha}-p_{j}$ for $1 \rightarrow 2$ splitting $\alpha \rightarrow(\alpha-j)+j$. Note that $\alpha$ and $\alpha-j$ are sets of parton indices while $j$ is the index of a single parton. This formula can apply 
generally, with the massless case restored by setting the parton masses $m_{\alpha}, m_{j}$, and $m_{\alpha-j}$ to zero. For final-state parton shower (FSPS), the virtuality of the parent parton can be reconstructed from those of the child partons, in a recursive way which is in the same direction as jet clustering. This procedure traces back to the partons at the end of the parton shower chains, which are physical particles and hence on-shell, $p_{j}^{2}=m_{j}^{2}$. In this way, the virtualities of all partons can be reconstructed.

In addition to parton masses, which are known, and virtualities that need to be reconstructed, there are two parameters, the evolution scale $t$, which takes the same role as time in the decay process of an unstable particle, and the energy fraction $z$, which is an analogy of energy partitioning between the decay products,

$$
z \equiv \frac{E_{j}}{E_{\alpha}}=\frac{E_{\alpha}-E_{\alpha-j}}{E_{\alpha}}
$$

taken away by one of the two child partons. ${ }^{1}$ In other words, the parton shower is controlled by these two parameters, $t$ and $z$. The evolution scale $t$ is related to the transverse momentum of the child partons,

$$
\left.t \equiv \frac{P_{\alpha}^{2}-m_{\alpha}^{2}}{z(1-z)}\right|_{P_{j}^{2}=m_{j}^{2}, P_{\alpha-j}^{2}=m_{\alpha-j}^{2}}=\frac{1}{z(1-z)}\left[\frac{m_{j}^{2}}{z}+\frac{m_{\alpha-j}^{2}}{1-z}+\frac{\mathbf{p}_{\perp j}^{2}}{z(1-z)}-m_{\alpha}^{2}\right] .
$$

For FSPS, the evolution scale $t$ and virtualities are positive. An immediate conclusion is that the parent parton has larger virtuality than the child partons. Consequently, $P_{\alpha}^{2}$ increases when clustering, which is the reverse of parton shower. Nevertheless, parton shower tends to emit soft partons, $z \rightarrow 0$. By clustering the child parton $j$, the relative energy increase from $E_{\alpha-j}$ to $E_{\alpha}$ is proportional to $z$ as defined in (2.2). On the other hand, the relative increase in the second term of (1.1) is suppressed even more, by a factor of,

$$
\frac{1}{E_{\alpha}}\left[\frac{P_{\alpha}^{2}-m_{\alpha}^{2}}{E_{\alpha}}-\frac{P_{\alpha-j}^{2}-m_{\alpha-j}^{2}}{E_{\alpha-j}}\right]=\frac{p_{j}^{2}-m_{j}^{2}}{z E_{\alpha}^{2}}+z(1-z) \frac{t}{E_{\alpha}^{2}} .
$$

Since the parton masses are very small, they can be omitted for convenience, $\left(P_{\alpha}^{2}-\right.$ $\left.m_{\alpha}^{2}\right) / E_{\alpha} \approx P_{\alpha}^{2} / E_{\alpha}$. For a soft emission, the parton with index $j$ tends to be a finalstate particle, $p_{j}^{2}-m_{j}^{2} \rightarrow 0$, making the first term vanish. In addition, the evolution scale $t$ decreases much faster than energy because of angular ordering [17, 18], appears as $t_{i}<\left(1-z_{i-1}\right)^{2} t_{i-1}$ for FSPS, with the indices assigned according to the sequence of splittings in parton shower, the smaller the earlier. Note that $t_{i}$ has very small chance of being close to the starting scale $\left(1-z_{i-1}\right)^{2} t_{i-1}$ due to suppression by the so-called Sudakov factor $\Delta(t)$, which is an analogy of the exponential decrease, $e^{-\Gamma t}$, in particle decay. Consequently, the order of the second term in (2.4) is lower than $\mathcal{O}(z)$, and the increase in $P_{\alpha}^{2} / E_{\alpha}$ is expected to be smaller than the increase in $E_{\alpha}$. In total, the expression inside

\footnotetext{
${ }^{1}$ For convenience, we have adopted the parton shower notation of energy fraction, which is different from the original notation $E_{j} / E_{\alpha} \equiv r_{j}$ used in [14] where $z$ is used to denote the angle between $\mathbf{P}_{\alpha}$ and $\mathbf{p}_{j}, \cos \theta$, instead.
} 
the parenthesis of (1.1) is roughly constant. This can be made apparent in the expanded form,

$$
J_{\beta}\left(P_{\alpha}\right)=E_{\alpha}\left[(1-\beta)+\beta v_{\alpha}^{2}\right] .
$$

as a function of the jet velocity $v_{\alpha} \equiv\left|\mathbf{P}_{\alpha}\right| / E_{\alpha}$, which does not change much. For an energetic shower, the partons are highly relativistic, $v_{\alpha} \approx 1$. Nevertheless, $v_{\alpha}$ can have slight decrease by clustering since $P_{\alpha}^{2} / E_{\alpha}^{2}=1-v_{\alpha}^{2}$ increases as indicated by (2.4). The jet function $J_{\beta}$ increases when reversing the parton shower chain, mainly because of the increase in the clustering scale $E_{\alpha}$, and hence can serve as a natural measure for reconstructing the parton shower history.

In the Georgi algorithms, one extra requirement is that, the jet function $J_{\beta}\left(P_{\alpha}\right)$ is positive, imposing a constraint on the jet velocity [14],

$$
v_{\min }^{2} \equiv 1-\frac{1}{\beta} \leq v^{2} \leq 1 .
$$

Note that $v_{\min }^{2}$ is just a notation. The only constraint is $v_{\min }^{2}$ should be smaller than 1 , otherwise, (2.6) would become meaningless. Equivalently, $\beta$ should be positive. Depending on the value of $\beta, v_{\min }^{2}$ can take any value, even negative values. Nevertheless, the value of $\beta$ should not be too large. Otherwise, $1-1 / \beta \approx 1$, hence $v^{2} \approx 1$, rendering the allowed range of jet velocity to be highly suppressed and only almost time-like jets can have a positive jet function. For $\beta<1$, the whole range of jet velocity, $0 \leq v^{2} \leq 1$, can be covered by positive jet function.

\section{Clustering with massive subjets}

Jet algorithm is a tool to quantify and visualize the parton shower process. Hence, it is helpful to provide a geometrical picture of clustering. To achieve this, an intuitive choice is cone size. With energy and momentum magnitude fixed, those subjets contained within a certain cone are all clustered. This property has already been implicitly incorporated [14] in the jet definition (1.1) which only depends on the angle $\theta$ between the jet 3 -momentum $\mathbf{P}_{\alpha}$ and the 3-momentum $\mathbf{p}_{j}$ of the subjet,

$$
J_{\beta}\left(P_{\alpha}+p_{j}\right)=\left(E_{\alpha}+E_{j}\right)\left[(1-\beta)+\beta \frac{\left|\mathbf{P}_{\alpha}\right|^{2}+2\left|\mathbf{P}_{\alpha}\right|\left|\mathbf{p}_{j}\right| \cos \theta+\left|\mathbf{p}_{j}\right|^{2}}{\left(E_{\alpha}+E_{j}\right)^{2}}\right] .
$$

The jet clustering criterion that the jet function increases, $J_{\beta}\left(P_{\alpha}+p_{j}\right)>J_{\beta}\left(P_{\alpha}\right)$, constrains the inclusion cone size, as will be explored in detail below.

Before diving into details, let us first take a look at the big picture and see what properties we should expect the jet function to have, from logical consistency and the property of parton shower, whose structure we want to describe by jet clustering. First, the result of jet clustering should be independent of the clustering sequence and hence logically consistent. For two subjets with the same energy $E_{j}$, the same 3 -momentum magnitude $\left|\mathbf{p}_{j}\right|$, and the same angle $\theta$ with respect to the jet 3 -momentum $\mathbf{P}_{\alpha}$, both of them should be clustered if one of them is. Otherwise, one is clustered while the other is not, leaving the result process-dependent for a sequential clustering. In other words, the 


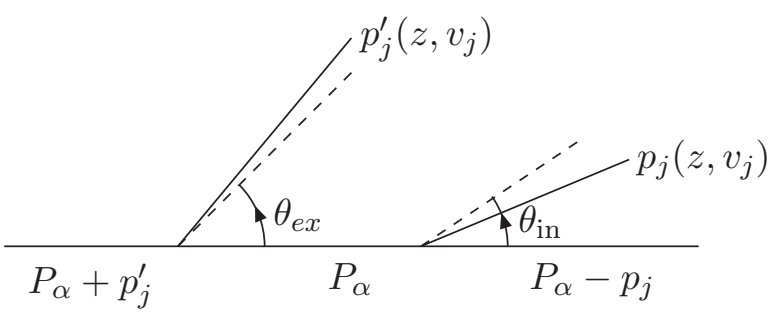

Figure 1. The inclusion $\left(\theta_{\text {in }}\right)$ and exclusion $\left(\theta_{e x}\right)$ cones (dashed lines) in the sequential clustering of two subjets $p_{j}$ and $p_{j}^{\prime}$ (solid lines) with the same energy $z$ and momentum magnitude $v_{j}$.

cone size should not shrink after swallowing a subjet [14]. What has not been revealed in [14] is the fact that this property is also consistent with angular ordering [17, 18], which claims that the opening angle between child partons keeps decreasing during parton shower. When reversing the process with jet clustering, the inclusion cone size should increase in order to accommodate all partons branching from the same chain. Hence, this first property is actually a requirement by parton shower, not just by logical consistency. It has been depicted in figure 1 as a sequential clustering of two subjets with the same energy, parametrized by $z$, and momentum magnitude, denoted as jet velocity $v_{j}$. We need to compare the two cones in two sequential clustering steps, defining the cone at the first as inclusion cone, $\theta_{\text {in }}$, and the second as exclusion cone, $\theta_{e x}$. The exclusion cone should not be smaller than the inclusion cone, $\theta_{e x} \geq \theta_{\text {in }}$. The second property comes from the tendency of parton shower to emit softer parton at earlier stage with larger opening angle $[15,16]$. Consequently, to make jet clustering approach the real parton shower process, it is necessary to have a larger cone size for softer subjet. Although it has been noticed in [14] that "the bound on jet 'size' in the sense of the largest possible angle of a particle in the jet from the jet direction is determined by the soft particles in the jet", it is only from parton shower that we realize this is a "must". We will show that these first two properties can be parameterized with a same quantity. Together, they eliminate the parameter region, $\beta<0$. The third property is that the inclusion cone cannot be too large. For the simplest case of $e^{+} e^{-} \rightarrow j j$ at LEP, the inclusion cone should not be larger than half sphere. Otherwise, the two jets cannot be separated. We will show that this gives a more stringent limit, $\beta>1$.

In the pioneering work [14], the author claims that "in practice we will typically be interested in masses $\sqrt{p_{j}^{\mu} p_{j \mu}}$ that are small compared to their energies and can be ignored in leading order". As pointed out therein, "this is not necessary for the construction, but it leads to considerable simplification". We will show that it needs not to ignore the parton mass $\sqrt{p_{j}^{\mu} p_{j \mu}}$. In this section, we derive the most general form of the Georgi algorithms [14] by keeping the subjet velocity $v_{j} \equiv\left|\mathbf{p}_{j}\right| / E_{j}$ without simplification. The massless case can be restored by when $v_{j}$ approaches 1 .

\subsection{The inclusion cone}

Let us start with the clustering of the first subjet with momentum $p_{j}$ of figure 1 to determine the inclusion cone size in terms of kinematic variables and possible constraints. 
For convenience of comparison with the clustering of the second subjet $p_{j}^{\prime}$, which will be explored in section 3.2 , we parameterize the clustering criterion on a common ground $P_{\alpha}$ where $p_{j}$ has already been clustered, but $p_{j}^{\prime}$ has not. In other words, $p_{j}$ is a part of $P_{\alpha}$, to be exact $j \in \alpha$ where $\alpha$ is a set of subjet indices, and the jet momentum before clustering is $P_{\alpha}-p_{j}$. Jet clustering criterion requires that the jet function (1.1) increases,

$$
J_{\beta}\left(P_{\alpha}\right)>\max \left\{J_{\beta}\left(P_{\alpha}-p_{j}\right), J_{\beta}\left(p_{j}\right)\right\} .
$$

Note that the jet function increases with respect to both subjets, because in reality it is impossible to distinguish the two sources. Using the expanded form (2.5) of the jet function, these two constraints can be expressed as,

$$
\begin{aligned}
& (1-\beta)+\beta v_{\alpha}^{2}>(1-z)\left[(1-\beta)+\frac{\beta}{(1-z)^{2}}\left(v_{\alpha}^{2}+z^{2} v_{j}^{2}-2 z \cos \theta v_{\alpha} v_{j}\right)\right], \\
& (1-\beta)+\beta v_{\alpha}^{2}>z\left[(1-\beta)+\beta v_{j}^{2}\right] .
\end{aligned}
$$

We can see that the second inequality (3.3b) gives a limit on the clustered jet velocity,

$$
v_{\alpha}^{2}-v_{\min }^{2}>z\left(v_{j}^{2}-v_{\min }^{2}\right) .
$$

This simply indicates that if $J_{\beta}\left(p_{j}\right)$ is positive, $J_{\beta}\left(P_{\alpha}\right)$ is also positive. The jet velocity range is enlarged after clustering for $\beta>1, v_{\alpha}^{2}$ is even closer to $v_{\min }^{2}$ than $v_{j}^{2}$, due to the $z$ factor in (3.4) which originates from the enhancement contributed by the clustering scale $E_{\alpha}$ in the jet definition (1.1).

On the other hand, (3.3a) limits the jet cone size,

$$
\cos \theta>\cos \theta_{\mathrm{in}} \equiv \frac{\left[(1-z) v_{\min }^{2}+z v_{j}^{2}\right]+v_{\alpha}^{2}}{2 v_{\alpha} v_{j}} \geq \sqrt{(1-z) \frac{v_{\min }^{2}}{v_{j}^{2}}+z} .
$$

Note that the second inequality comes from minimizing $\cos \theta_{\text {in }}$ as a function of $v_{\alpha}$, and the equality happens on the boundary (3.4) of $v_{\alpha}$ if $\beta>1$. For massive subjet, $v_{j}^{2}<1$, the maximal inclusion cone is smaller than the massless limit. The most interesting feature is the dependence on the energy fraction $z$. We can decompose the cone size $\cos \theta_{\text {in }}(3.5)$ as a series of $z$,

$$
\cos \theta_{\text {in }} \equiv \frac{1}{2 v_{\alpha} v_{j}}\left[\left(v_{\alpha}^{2}+v_{\min }^{2}\right)+\left(v_{j}^{2}-v_{\min }^{2}\right) z\right],
$$

which reduces to the Eq. (15) of [14] in the massless limit, $v_{j} \rightarrow 1$, replacing $v_{\min }^{2}$ with $1-1 / \beta$, while changing the notations, $z \rightarrow r_{j}$ and $\cos \theta_{\text {in }} \rightarrow z$. This indicates that the cone increases with decreasing $z$. In other words, the cone is larger for softer subjet if (2.6) is satisfied, which is exactly what required by parton shower. It provides a strong support for the requirement on the positiveness of the jet function.

The inclusion region (3.6) can also be expressed in terms of $\sin \left(\theta_{\text {in }} / 2\right)$ which increases with the cone size,

$$
2 \sin ^{2}\left(\frac{1}{2} \theta_{\mathrm{in}}\right)=\frac{1}{2 v_{\alpha} v_{j}}\left[(1-z)\left(v_{j}^{2}-v_{\min }^{2}\right)-\left(v_{\alpha}-v_{j}\right)^{2}\right]>\frac{v_{j}-v_{\alpha}}{v_{j}},
$$


where the inequality comes from (3.4). Since virtuality increases when reversing the parton shower history according to (2.1), the velocity decreases, $v_{j}>v_{\alpha}$. This indicates that the inclusion cone cannot be too small. If we take the soft and massless limits, $z \rightarrow 0$ and $v_{j}, v_{\alpha} \rightarrow 1$ respectively, the inclusion cone becomes $\cos \theta_{\text {in }}=\left(1+v_{\min }^{2}\right) / 2$, which together with (2.6) reduces to the eq. (17) of [14], $\theta_{\text {in }}=2 \arcsin (1 / 2 \sqrt{\beta})$. On the other hand, in the soft limit, $z \rightarrow 0$, massless limit for the subjet, $v_{j} \rightarrow 1$, and the lower limit (2.6) on the jet velocity after clustering, $v_{\alpha}^{2}=v_{\min }^{2}=1-1 / \beta$, it reduces to $\cos \theta_{\text {in }}=v_{\min }=v_{\alpha}$ and equivalently, $\theta_{\text {in }}=2 \arcsin \sqrt{(1-\sqrt{1-1 / \beta}) / 2}$, which is the eq. (18) of [14].

\subsection{The exclusion cone}

After including the first subjet $p_{j}$ with certain energy $(z)$ and 3-momentum magnitude $\left(v_{j}\right)$, the jet momentum changes from $P_{\alpha}-p_{j}$ to $P_{\alpha}$, as shown in figure 1 . This leads to modification of the cone size which we will try to derive here. The result is compared with the one of inclusion cone. If the jet clustering is self-consistent, a second subjet $p_{j}^{\prime}$ with the same energy $z$ and 3-momentum magnitude $v_{j}$ should also be clustered if inside the inclusion region (3.5). In addition, the largest cone size is established in terms of the jet parameter $\beta$.

Suppose this second subjet $p_{j}^{\prime}$ can not be clustered, namely, the jet function (1.1) decreases if so,

$$
J_{\beta}\left(P_{\alpha}\right)>\max \left\{J_{\beta}\left(P_{\alpha}+p_{j}^{\prime}\right), J_{\beta}\left(p_{j}^{\prime}\right)\right\} .
$$

From these two constraints we can derive the exclusion cone, parametrized with $\theta_{e x}$ as shown if figure 1. Using the expanded form (2.5) of the jet function, we can get two inequalities,

$$
\begin{aligned}
& (1-\beta)+\beta v_{\alpha}^{2}>(1+z)\left[(1-\beta)+\frac{\beta}{(1+z)^{2}}\left(v_{\alpha}^{2}+z^{2} v_{j}^{2}+2 z \cos \theta^{\prime} v_{\alpha} v_{j}\right)\right], \\
& (1-\beta)+\beta v_{\alpha}^{2}>z\left[(1-\beta)+\beta v_{j}^{2}\right]
\end{aligned}
$$

Note that $(3.9 \mathrm{~b})$ is exactly $(3.3 \mathrm{~b})$, leading to the same constraint (3.4) on $v_{\alpha}^{2}$. But the cone boundary,

$$
\cos \theta^{\prime}<\cos \theta_{e x} \equiv \frac{\left[(1+z) v_{\min }^{2}-z \beta v_{j}^{2}\right]+v_{\alpha}^{2}}{2 v_{\alpha} v_{j}},
$$

is different from the inclusion cone boundary $\cos \theta_{\text {in }}$ in (3.5). This difference can be traced back to the different signs of $p_{j}$ and $p_{j}^{\prime}$ in the jet functions $J_{\beta}\left(P_{\alpha}-p_{j}\right)$ and $J_{\beta}\left(P_{\alpha}+p_{j}^{\prime}\right)$, respectively, leading to an effective replacement, $z \rightarrow-z$. Note that the inclusion cone (3.5) and the exclusion cone (3.10) are well separated due to the lower limit (2.6) on $v_{j}$,

$$
\cos \theta_{\text {in }}-\cos \theta_{e x}=\frac{z}{v_{\alpha} v_{j}}\left(v_{j}^{2}-v_{\min }^{2}\right)>0 .
$$

The subjet within the inclusion cone (3.5) with the same energy $z$ and 3-momentum magnitude $v_{j}$ can be readily clustered. Imposing this property eliminates the possibility of $v_{\min }^{2}>1$, or equivalently $\beta<0$, since the jet velocity is bounded by the speed of light from above, $v_{j}^{2} \leq 1$. 
It should be emphasized that the only difference between the inclusion cone (3.5) and the exclusion cone (3.10) is a sign difference associated with $z$. For soft jet, $z \rightarrow 0$, the difference between the two cones actually also characterizes the ability of accommodating softer subjet. This can be explicitly seen by comparing the expression of $\cos \theta_{\text {in }}-\cos \theta_{\text {ex }}$ in (3.11) and the linear term of $z$ in (3.6) whose coefficients differ by only a factor of 2 . The inclusion region should expand during the jet clustering process in order to accommodate softer subjet while it is the opposite for the exclusion region, approaching each other. This is consistent with the observation in [14] that, "the particles not in the jet can only approach the jet boundary as $r_{j} \rightarrow 0$ ".

Similar to (3.7), the exclusion cone size is bounded by,

$$
2 \sin ^{2}\left(\frac{1}{2} \theta_{e x}\right)=\frac{1}{2 v_{\alpha} v_{j}}\left[(1+z)\left(v_{j}^{2}-v_{\min }^{2}\right)-\left(v_{\alpha}-v_{j}\right)^{2}\right]<1-\frac{v_{\min }^{2}}{v_{\alpha} v_{j}},
$$

where the inequality comes from (3.4). A direct consequence is,

$$
2 \sin ^{2}\left(\frac{1}{2} \theta_{e x}\right)<1-v_{\min }^{2}=\frac{1}{\beta}
$$

since $v_{\alpha}, v_{j} \leq 1$. For $\beta \geq 1$, the exclusion cone is smaller than half sphere, $\theta_{e x}<90^{\circ}$. Take a two-jet event in the center-of-mass frame as illustration, for example $e^{+} e^{-} \rightarrow j j$ at LEP, jet-clustering should reconstruct two jets that are back-to-back. On the other hand, the two jets can be mixed with each other if $\beta<1$, rendering the cone to be larger than half sphere, which is a not good choice. This observation can serve as a guide for choosing a reasonable value for $\beta$. To recognize an event with more primary jets, $\beta$ should be larger. Note that this limit is independent of $z$.

\section{Generalized jet function}

In the previous section, we have shown that the jet parameter $\beta$ is closely related cone size cutoff. With larger $\beta$, the cone size becomes smaller. It would be a good practice to find an extension, providing more degrees of freedom when choosing the cone size cutoff. Here, we try to develop a possible generalization by introducing jet function index $n$, which is also related to cone size as we will elaborate in the remaining part of this section.

Technically speaking, the generalization comes from the observation that $v_{\alpha}^{2}$ appears on both sides of (3.3a) and (3.9a), where it is possible to make a complete cancellation of the $v_{\alpha}^{2}$ terms if the prefactor $1-z$ is replaced by $(1-z)^{2}$. The same trick can be used to remove the factor $1-\beta$ in (3.3) and (3.9) by eliminating the prefactor $1-z$. Nevertheless, the first observation can become true but the latter is not realistic as will be shown in detail below.

Since the power of the $1-z$ prefactor can be traced back to the power of the clustering scale $E_{\alpha}$, to achieve the small tricks we need to generalize the jet function (1.1) as follows,

$$
J_{\beta}^{(n)}\left(P_{\alpha}\right) \equiv E_{\alpha}^{n}\left(1-\beta \frac{P_{\alpha}^{2}}{E_{\alpha}^{2}}\right)=E_{\alpha}^{n}\left[(1-\beta)+\beta v_{\alpha}^{2}\right]
$$


with an extra jet index $n$. Accordingly, the jet function (1.1) can be renamed as $J_{\beta}\left(P_{\alpha}\right) \equiv$ $J_{\beta}^{(1)}\left(P_{\alpha}\right)$. For generality, we keep the jet function index $n$ as a free parameter in the following derivations. Note that $n$ needs not to be an integer and can serve as a jet function parameter as $\beta$. Its value is constrained by kinematics. As we have argued that the jet function increases mainly because of the increase in the prefactor $E_{\alpha}^{n}$. The jet function index $n$ cannot be arbitrarily small for the jet function to increase fast enough. At least, $n$ has to be positive. We will show further constraints in the following analysis.

With this generalized jet definition, the limit (2.6) on jet velocity from the requirement that the jet function has to be positive is still the same. As expected, the $1-z$ and $z$ prefactors in the inclusion (3.3) and exclusion (3.9) criteria receives a nontrivial power $n$,

$$
\begin{aligned}
& (1-\beta)+\beta v_{\alpha}^{2}>(1 \mp z)^{n}\left[(1-\beta)+\frac{\beta}{(1 \mp z)^{2}}\left(v_{\alpha}^{2}+z^{2} v_{j}^{2} \mp 2 z \cos \theta v_{\alpha} v_{j}\right)\right], \\
& (1-\beta)+\beta v_{\alpha}^{2}>z^{n}\left[(1-\beta)+\beta v_{j}^{2}\right]
\end{aligned}
$$

with the sign $\mp$ corresponding to inclusion and exclusion cones, respectively. From the second inequality, we can get a generalized form of the jet velocity constraint (3.4),

$$
v_{\alpha}^{2}-v_{\min }^{2}>z^{n}\left(v_{j}^{2}-v_{\min }^{2}\right) .
$$

Similarly, $v_{\alpha}^{2}$ is contained within the positive jet function region (2.6) if $v_{j}^{2}$ already satisfies it. The jet velocity range of the $\alpha$-set becomes larger than that of the subjet, due to suppression from the prefactor $z^{n}$. In soft jet approximation, $z \rightarrow 0$, the difference can be significant.

The inclusion cone (3.5) and the exclusion cone (3.10) are constrained by the first inequality $(4.2 \mathrm{a})$,

$$
\begin{aligned}
\cos \theta_{\text {in }}^{(n)} & \equiv \frac{1}{2 z v_{\alpha} v_{j}}\left\{\left[1-(1-z)^{2-n}\right]\left(v_{\alpha}^{2}-v_{\min }^{2}\right)+z^{2}\left(v_{j}^{2}-v_{\min }^{2}\right)+2 z v_{\min }^{2}\right\}, \\
\cos \theta_{e x}^{(n)} & \equiv \frac{1}{2 z v_{\alpha} v_{j}}\left\{\left[(1+z)^{2-n}-1\right]\left(v_{\alpha}^{2}-v_{\min }^{2}\right)-z^{2}\left(v_{j}^{2}-v_{\min }^{2}\right)+2 z v_{\min }^{2}\right\} .
\end{aligned}
$$

Then, we can explore the difference between them,

$$
\begin{aligned}
\cos \theta_{\text {in }}^{(n)}-\cos \theta_{e x}^{(n)} & =\frac{2-(1-z)^{2-n}-(1+z)^{2-n}}{2 z v_{\alpha} v_{j}}\left(v_{\alpha}^{2}-v_{\min }^{2}\right)+\frac{z}{v_{\alpha} v_{j}}\left(v_{j}^{2}-v_{\min }^{2}\right) \\
& \geq \frac{1}{2 v_{\alpha} v_{j}}\left\{z^{n-1}\left[2-(1-z)^{2-n}-(1+z)^{2-n}\right]+2 z\right\}\left(v_{j}^{2}-v_{\min }^{2}\right)
\end{aligned}
$$

If the clustering algorithm is self-consistent, the inclusion cone expands after clustering a subjet with the same energy and 3-momentum magnitude with the only difference in opening angle. This property makes itself explicit as the inequality in (4.5), which is satisfied for $n \leq 2$. The self-consistency requirement of jet clustering provides an upper limit on the jet function index $n$. Note that, for both $n=1$ and $n=2,(4.5 \mathrm{~b})$ reduces to (3.11). 
Now let us take a look at the soft region. If the subjet is soft, $z \rightarrow 0$, the inclusion and exclusion cones (4.4) can be approximated by an expansion up to the linear order of $z$,

$$
\begin{aligned}
& \cos \theta_{\text {in }}^{(n)} \approx \frac{1}{2 v_{\alpha} v_{j}}\left\{(2-n)\left(1-\frac{1-n}{2} z\right)\left(v_{\alpha}^{2}-v_{\min }^{2}\right)+z\left(v_{j}^{2}-v_{\min }^{2}\right)+2 v_{\min }^{2}\right\}, \\
& \cos \theta_{e x}^{(n)} \approx \frac{1}{2 v_{\alpha} v_{j}}\left\{(2-n)\left(1+\frac{1-n}{2} z\right)\left(v_{\alpha}^{2}-v_{\min }^{2}\right)-z\left(v_{j}^{2}-v_{\min }^{2}\right)+2 v_{\min }^{2}\right\} .
\end{aligned}
$$

The difference (4.5) between the inclusion and exclusion cones is roughly,

$$
\cos \theta_{\mathrm{in}}^{(n)}-\cos \theta_{e x}^{(n)} \approx-\frac{1}{2 v_{\alpha} v_{j}}(2-n)(1-n) z\left(v_{\alpha}^{2}-v_{\min }^{2}\right)+\frac{z}{v_{\alpha} v_{j}}\left(v_{j}^{2}-v_{\min }^{2}\right),
$$

which is highly suppressed. Nevertheless, overlapping can still happen. To avoid this tiny chance, the following relation between $v_{\alpha}^{2}$ and $v_{j}^{2}$ has to be satisfied,

$$
v_{j}^{2}-v_{\min }^{2} \geq \frac{(2-n)(1-n)}{2}\left(v_{\alpha}^{2}-v_{\min }^{2}\right) .
$$

Together with (4.3), we can get,

$$
\left[\frac{1}{z^{n}}-\frac{(2-n)(1-n)}{2}\right]\left(v_{\alpha}^{2}-v_{\min }^{2}\right) \geq 0,
$$

which is always true for $n>0$. The jet-clustering self-consistency in the soft region also imposes a lower limit on the jet function index $n$. Since self-consistency in the soft region is directly related to the requirement that soft emission has a larger inclusion cone, in order to make the jet algorithm approach the parton shower evolution, this lower limit can also be treated as a requirement of the second property.

To see the boundary on the exclusion cone, we need to first check the sign of $z$ in the expanded form (4.6b),

$$
\cos \theta_{e x}^{(n)} \approx \frac{1}{2 v_{\alpha} v_{j}}\left\{(2-n)\left(v_{\alpha}^{2}-v_{\min }^{2}\right)+2 v_{\min }^{2}+\left[\frac{(2-n)(1-n)}{2}\left(v_{\alpha}^{2}-v_{\min }^{2}\right)-\left(v_{j}^{2}-v_{\min }^{2}\right)\right] z\right\} .
$$

We can see that, by replacing $z$ with the help of (4.3) the exclusion cone can have a bound like (3.13), which is independent of $z$, as long as the coefficient of $z$ in (4.10) is negative. This can be guaranteed for $1 \leq n \leq 2$,

$$
\begin{aligned}
\cos \theta_{e x}^{(n)} \gtrsim & \frac{1}{2 v_{\alpha} v_{j}}\left[(2-n)\left(v_{\alpha}^{2}-v_{\min }^{2}\right)+2 v_{\min }^{2}\right] \\
& +\frac{1}{2 v_{\alpha} v_{j}}\left[\frac{(2-n)(1-n)}{2}\left(v_{\alpha}^{2}-v_{\min }^{2}\right)-\left(v_{j}^{2}-v_{\min }^{2}\right)\right]\left(\frac{v_{\alpha}^{2}-v_{\min }^{2}}{v_{j}^{2}-v_{\min }^{2}}\right)^{\frac{1}{n}} .
\end{aligned}
$$

This expression is a little bit too complicated, and we would try to make simplifications. Since the partons are quite relativistic, $v_{\alpha} \approx v_{j} \approx 1$, (4.11) reduces to,

$$
2 \sin ^{2}\left(\frac{1}{2} \theta_{e x}^{(n)}\right) \lesssim \frac{n(5-n)}{4} \frac{1}{\beta} .
$$


This simplification has another advantage of expressing the boundary in terms of the jet parameter $\beta$ and index $n$. The result (3.13) can be reproduced with $n=1$. We can see that both $\beta$ and $n$ are directly related to the kinematic boundary. For the cone size to be larger than half sphere, $\beta$ has a lower limit,

$$
\beta>\frac{4}{n(5-n)} \geq \frac{2}{3} .
$$

In the range $1 \leq n \leq 2$, the coefficient $n(5-n)$ decreases with $n$. Consequently, $\beta$ should increase with $n$. For the original scheme, $n=1, \beta>1$, leading to $0<v_{\min }^{2}<1$. Only part of the jet velocity range can be covered which is especially true with more than 2 jets and $\beta$ further enhanced. By generalization, $n>1$, the parameter $\beta$ can be smaller than 1 , leading to a negative $v_{\text {min }}^{2}$ which can cover the whole jet velocity range.

Similarly, there is a lower limit on the inclusion cone size,

$$
\begin{aligned}
\cos \theta_{\text {in }}^{(n)} \lesssim & \frac{1}{2 v_{\alpha} v_{j}}\left[(2-n)\left(v_{\alpha}^{2}-v_{\min }^{2}\right)+2 v_{\min }^{2}\right] \\
& -\frac{1}{2 v_{\alpha} v_{j}}\left[\frac{(2-n)(1-n)}{2}\left(v_{\alpha}^{2}-v_{\min }^{2}\right)-\left(v_{j}^{2}-v_{\min }^{2}\right)\right]\left(\frac{v_{\alpha}^{2}-v_{\min }^{2}}{v_{j}^{2}-v_{\min }^{2}}\right)^{\frac{1}{n}}
\end{aligned}
$$

which reduces to,

$$
2 \sin ^{2}\left(\frac{1}{2} \theta_{\mathrm{in}}^{(n)}\right) \gtrsim \frac{n(n-1)}{4} \frac{1}{\beta},
$$

in the relativistic limit. For $n \geq 1$, the inclusion cone cannot be arbitrarily small.

\section{$5 \quad$ Lorentz boost invariance}

From the constraint on the jet velocity (2.6), we can see that $\beta=1 /\left(1-v_{\min }^{2}\right)$ is actually the square of the corresponding boost factor $\gamma_{\min }=1 / \sqrt{1-v_{\min }^{2}}$. This indicates that $\beta$ has close relation with Lorentz boost. It is important to check how Lorentz boost affects the jet algorithm, especially for highly boosted jets at hadron collider like LHC.

The Lorentz boost can be represented by boost factor $\gamma_{B}$ and the direction of boosting. At each step of clustering, the jet momentum changes. Not just its magnitude is different, but also the direction. There is no uniform transformation on the jet momenta. For jet function under consideration, the jet mass, $P_{\alpha}^{2}$, is invariant, but the jet energy, $E_{\alpha}$, changes. The rate of energy change is different from jet to jet. This can be parametrized as,

$$
P_{\alpha}^{2} \rightarrow P_{\alpha}^{2}, \quad E_{\alpha} \rightarrow \gamma_{\alpha} E_{\alpha} .
$$

The rescaling factor $\gamma_{\alpha}$ is not universal, but varies from jet to jet as a function of the boost factor, $\gamma_{B}$, and the jet velocity, $v_{\alpha}$,

$$
\gamma_{\alpha} \equiv \gamma_{B}\left(1-\vec{v}_{B} \cdot \vec{v}_{\alpha}\right)
$$

where $\vec{v}_{B} \equiv \vec{P}_{B} / E_{B}$ is the velocity corresponding to the global Lorentz boost. Note that $\gamma_{\alpha}$ is not necessarily equal to $\gamma_{B}$. Only when the jet momentum $P_{\alpha}$ is perpendicular to the 
direction of the global Lorentz boost, the two Lorentz boost factors can be the same. The change in the dimensionless part of (4.1) can be compensated by,

$$
\beta \rightarrow \gamma_{\alpha}^{2} \beta,
$$

and the jet function should be redefined as,

$$
J_{\beta}^{(n)} \rightarrow \gamma_{\alpha}^{-n} J_{\gamma_{\alpha}^{2} \beta}^{(n)},
$$

to retain the original jet function value. Consequently, the clustering sequence remains. In this sense, the jet algorithm can be made Lorentz invariant.

\section{Conclusion}

We reveal the direct link between the Georgi algorithms of jet clustering and the parton shower kinematics. The energy increases when clustering jets, due to conservation, while the jet mass term $P_{\alpha}^{2} / E_{\alpha}$ does not increase much, because of the fact that parton shower tends to emit soft partons, $z \rightarrow 0$. Our observation provides a sound support for this elegant class of jet clustering algorithms whose kinematic features are explored in this work systematically for both massless and massive partons. We further generalize the jet function definition to $J_{\beta}^{(n)}\left(P_{\alpha}\right)$, with a free jet index $n$ which is constrained within the range $1 \leq n \leq 2$. Its upper limit comes from the logical consistency of the jet algorithm, while the lower from the requirement that the cone size cannot be arbitrarily large in order to avoid mixing up neighbor jets. The parameter $\beta$ and index $n$ have the meaning of phase space boundaries and are constrained as $\beta>4 / n(5-n) \geq 2 / 3$. In this generalization, the original Georgi algorithms can be recovered as special cases, $J_{\beta}\left(P_{\alpha}\right)=J_{\beta}^{(1)}\left(P_{\alpha}\right)$. Under Lorentz boost, the value of jet function at each step can be restored by adjusting $\beta$ and multiplying an overall factor $\gamma^{-n}$. In this sense, we claim that the jet algorithms can be made boost invariant.

\section{Acknowledgments}

SFG is grateful to Kaoru Hagiwara, Grisha Kirilin, and Junichi Kanzaki for discussions about parton shower and introduction to this field of research. The current work is supported by Grant-in-Aid for Scientific Research (No. 25400287) from JSPS. During the revision of this paper, the author received kind help from Prof. Howard Georgi to compare with his pioneering paper.

Open Access. This article is distributed under the terms of the Creative Commons Attribution License (CC-BY 4.0), which permits any use, distribution and reproduction in any medium, provided the original author(s) and source are credited.

\section{References}

[1] G.F. Sterman and S. Weinberg, Jets from Quantum Chromodynamics, Phys. Rev. Lett. 39 (1977) 1436 [INSPIRE]. 
[2] S. Catani, Y.L. Dokshitzer, M.H. Seymour and B.R. Webber, Longitudinally invariant $K_{t}$ clustering algorithms for hadron hadron collisions, Nucl. Phys. B 406 (1993) 187 [InSPIRE].

[3] S.D. Ellis and D.E. Soper, Successive combination jet algorithm for hadron collisions, Phys. Rev. D 48 (1993) 3160 [hep-ph/9305266] [INSPIRE].

[4] Y.L. Dokshitzer, G.D. Leder, S. Moretti and B.R. Webber, Better jet clustering algorithms, JHEP 08 (1997) 001 [hep-ph/9707323] [INSPIRE].

[5] M. Wobisch and T. Wengler, Hadronization corrections to jet cross-sections in deep inelastic scattering, hep-ph/9907280 [INSPIRE].

[6] M. Wobisch, Measurement and QCD analysis of jet cross-sections in deep inelastic positron proton collisions at $\sqrt{s}=300-G e V$, Rheinisch-Westfälischen Technischen Hochschule Aachen, DESI Thesis (2000), http://www-library.desy.de/cgi-bin/showprep.pl?desy-thesis00-049.

[7] M. Cacciari, G.P. Salam and G. Soyez, The Anti-k(t) jet clustering algorithm, JHEP 04 (2008) 063 [arXiv:0802.1189] [INSPIRE].

[8] S. Catani, Y.L. Dokshitzer, M. Olsson, G. Turnock and B.R. Webber, New clustering algorithm for multi - jet cross-sections in $e^{+} e^{-}$annihilation, Phys. Lett. B 269 (1991) 432 [INSPIRE].

[9] S. Moretti, L. Lönnblad and T. Sjöstrand, New and old jet clustering algorithms for electron - positron events, JHEP 08 (1998) 001 [hep-ph/9804296] [INSPIRE].

[10] G.C. Blazey et al., Run II jet physics, hep-ex/0005012 [INSPIRE].

[11] S.D. Ellis, J. Huston, K. Hatakeyama, P. Loch and M. Tonnesmann, Jets in hadron-hadron collisions, Prog. Part. Nucl. Phys. 60 (2008) 484 [arXiv:0712.2447] [InSPIRE].

[12] G.P. Salam, Towards Jetography, Eur. Phys. J. C 67 (2010) 637 [arXiv:0906.1833] [INSPIRE].

[13] A. Ali and G. Kramer, Jets and QCD: A Historical Review of the Discovery of the Quark and Gluon Jets and its Impact on QCD, Eur. Phys. J. H 36 (2011) 245 [arXiv:1012.2288] [INSPIRE].

[14] H. Georgi, A Simple Alternative to Jet-Clustering Algorithms, arXiv:1408.1161 [INSPIRE].

[15] S. Catani, L. Trentadue, G. Turnock and B.R. Webber, Resummation of large logarithms in $e^{+} e^{-}$event shape distributions, Nucl. Phys. B 407 (1993) 3 [INSPIRE].

[16] S. Gieseke, P. Stephens and B. Webber, New formalism for QCD parton showers, JHEP 12 (2003) 045 [hep-ph/0310083] [INSPIRE].

[17] A.H. Mueller, On the Multiplicity of Hadrons in QCD Jets, Phys. Lett. B 104 (1981) 161 [INSPIRE].

[18] B.I. Ermolaev and V.S. Fadin, Log - Log Asymptotic Form of Exclusive Cross-Sections in Quantum Chromodynamics, JETP Lett. 33 (1981) 269 [INSPIRE]. 